Une déclaration d'un comité consultatif (DCC)

Comité consultatif de la médicine tropicale et de la médecine des voyages (CCMTMV) ${ }^{\dagger}$

\title{
DÉCLARATION RELATIVE AUX MESURES DE PROTECTION INDIVIDUELLE POUR PRÉVENIR LES PIQÛRES OU MORSURES D'ARTHROPODES
}

\section{Préambule}

Le Comité consultatif de la médecine tropicale et de la médecine des voyages (CCMTMV) fournit à l'Agence de la santé publique du Canada (ASPC), de façon continue et en temps utile, des conseils de nature médicale, scientifique et de santé publique concernant les maladies tropicales infectieuses et les risques pour la santé associés aux voyages à l'étranger. L'Agence reconnaît que les conseils et les recommandations figurant dans cette déclaration reposent sur la pratique médicale et les connaissances scientifiques les plus à jour, et les diffuse dans le but d'informer les voyageurs ainsi que les professionnels de la santé qui sont appelés à leur prodiguer des soins.

Les personnes qui administrent ou utilisent des médicaments, des vaccins ou d'autres produits devraient bien connaître le contenu des monographies de produit ou des autres normes ou modes d'emploi approuvés. Les recommandations relatives à l'usage des produits et les autres renseignements présentés ici peuvent différer de ceux qui figurent dans la monographie ou toute autre norme ou instruction approuvée et pertinente établie par les fabricants autorisés. Rappelons que l'approbation demandée par les fabricants pour leurs produits, avec démonstration de leur innocuité et de leur efficacité à l'appui, ne s'applique qu'aux utilisations conformes à la monographie ou autre norme ou mode d'emploi approuvé.

Membres : Dr A. McCarthy (Président); Dr A.K. Boggild; Dr J. Brophy; Dr Y. Bui; Dr C. Greenaway; Dr M. Crockett; Dr W. Ghesquiere; Mme A. Henteleff; Dr M. Libman; Dr K.L. McClean; Dr P.J. Plourde; Dr P. Teitelbaum.

Représentants d'office : Dr P. Charlebois; Dr P. McDonald; Dr S. Schofield; Dr M. Tepper

Représentants de liaison : Dr G. Brunette; Dr C. Hui.

Membre émérite : Dr C.W.L. Jeanes.

†Le présent document a été préparé par S. Schofield et P. Plourde et approuvé par le CCMTMV.

Le CCMTMV remercie sincèrement l'Agence de réglementation de la lutte antiparasitaire, Organisation mondiale de la Santé Programme d'évaluation des pesticides (WHO Pesticide Evaluation Scheme: WHOPES), et le Conseil des Forces armées de la lutte antiparasitaire pour avoir contribué à l'élaboration de la déclaration. 


\section{Introduction}

Divers pathogènes sont transmis à l'humain par des arthropodes vecteurs, dont certains sont la cause de maladies graves associées aux voyages (p. ex., le paludisme et la dengue). Habituellement, mais pas toujours (comme dans le cas de la maladie de Chagas et du typhus exanthématique), la transmission se produit par la piqûre ou la morsure d'un vecteur, alors que les pathogènes en cause terminent en partie leur cycle de vie dans l'organisme de l'arthropode.

Bien que les moustiques soient les vecteurs les plus notoires et les plus importants de maladies associées aux arthropodes (MAA), plusieurs autres arthropodes peuvent transmettre des pathogènes (p. ex., les tiques, les phlébotomes, les tabanidés, les poux, les acariens, les puces, les mouches tsé-tsé et les réduves) (voir l'annexe 1). Leur diversité peut compliquer les efforts de prévention des MAA et leur traitement, car non seulement plusieurs maladies sont-elles en cause, mais l'éventail des vecteurs est aussi vaste que les comportements et les épidémiologies qui leur sont associés. Cette complexité est bien illustrée par le contraste entre deux groupes de moustiques, des genres Aedes et Anopheles, lesquels sont responsables de la transmission de la dengue et du paludisme, respectivement. Bien que ces groupes de moustiques se ressemblent à première vue, chacun a évolué de son côté depuis plus de 100 millions d'années (1) et ont donc eu amplement le temps de mettre au point différents cycles biologiques et stratégies de survie. Par conséquent, d'une part, il y a l'Aedes aegypti, le principal vecteur de la dengue (2), qui vit généralement à l'intérieur et à proximité des zones aménagées et qui pique habituellement le jour, quoique plus souvent le matin et à l'approche de la nuit. D'autre part, il y a l'Anopheles gambiae s.str., que l'on trouve en Afrique et qui est le vecteur par excellence du paludisme. Cette espèce habite essentiellement les zones rurales et a tendance à piquer dans les intérieurs et dans l'obscurité. Alors que les deux espèces s'alimentent du sang des hôtes humains et transmettent des pathogènes importants, c'est surtout ce qui les distingue qui importe quant au moment de la journée et aux endroits où il y a risque de contagion. Cette diversité ne se limite pas aux différents genres de moustiques ni aux classes d'insectes. En effet, même chez les quarante et quelques principales espèces de vecteurs du paludisme (3), il y a des différences significatives et pertinentes aux interventions qui reposent sur le comportement et les caractéristiques biologiques. Par exemple, I'A. gambiae s.str. susmentionné tend à être endophage, c'est-à-dire qu'il pique à l'intérieur des habitations. Cette propension, en conjonction avec ses habitudes nocturnes, signifie que les moustiquaires de lit constituent une mesure incontournable (4). Par contre, l'A. albimanus, un vecteur important dans les Amériques, pique souvent à l'extérieur des habitations et en début de soirée, avant que les gens se mettent au lit. Par conséquent, les moustiquaires de lit, tout en étant importantes, le sont relativement moins contre cette espèce, du moins en comparaison avec les insectifuges topiques et le port de vêtements appropriés. Pour compliquer les choses, les données sur cette dernière espèce semblent indiquer qu'elle a une « tolérance » accrue à l'insectifuge DEET (N,N-diéthyl-3-méthylbenzamide, aussi connu sous I'appellation $\mathrm{N}, \mathrm{N}$-diéthyl-m-toluamide) de sorte que la durée de la protection est écourtée (5).

L'essentiel du message à retenir par le médecin clinicien et le voyageur, c'est que les caractéristiques de chacun des vecteurs varient beaucoup et qu'il faut, dans la mesure du possible, adapter les approches prophylactiques en tenant compte de ce fait. Cela pourrait être aussi simple que d'appliquer à nouveau de l'insectifuge lorsqu'on remarque la présence de piqûres au lieu de s'en tenir aux prescriptions indiquées sur l'étiquette ou en se protégeant avec une moustiquaire de lit au moment de se reposer, indépendamment de l'heure du jour.

\section{Mesures de protection individuelle}

Compte tenu de la vaste diversité des vecteurs, on peut s'étonner du fait qu'une seule approche (c.-à-d. la prévention des piqûres et des morsures) fonctionne pour tous les différents genres et que, par ailleurs, une petite série d'interventions (c.-à-d. des mesures de protection individuelle [MPI]) soit si utile à cet égard. Ceci contraste de façon marquée avec d'autres interventions prophylactiques, comme la vaccination et la chimioprophylaxie, dont l'efficacité réelle se limite souvent à un seul pathogène pour prévenir une maladie précise. 
Cette déclaration présente des directives et formule des recommandations sur I'utilisation de MPI permettant d'éviter des piqûres et des morsures d'arthropodes. Ces interventions ont été appliquées depuis la nuit des temps dans les sociétés traditionnelles (6; 7), avec des approches modernes évoluant au rythme de la recherche, du développement et des découvertes depuis le dernier siècle, souvent le résultat d'innovations subventionnées par les forces militaires (8). Bien que plusieurs types de MPI ont probablement certains avantages au plan de la protection (9), la présente déclaration porte avant tout sur les barrières physiques (p. ex., vêtements, moustiquaires) et chimiques (p. ex., insectifuges topiques et traitement des moustiquaires). Cet accent tient compte, premièrement, d'une stratégie permettant de gérer le niveau de l'effort requis d'élaboration de lignes directrices et, deuxièmement, de l'opinion du comité selon lequel il vaut mieux privilégier des MPI qui répondent aux critères suivants :

- I'application des mesures relève d'abord du voyageur;

- I'accès est possible au Canada ou depuis le Canada;

- des données pertinentes, fiables et récentes sont disponibles et permettent de juger des bienfaits et des effets nocifs (p. ex., des insectifuges et des insecticides).

\section{Évaluation des preuves et recommandations}

Les preuves ont été évaluées en application de la déclaration de 1994 du CCMTMV sur l'approche de la médecine fondée sur les preuves (MFP) (annexe 2) (10). Le CCMTMV se prononce sur les points suivants au sujet du présent processus d'examen :

- On n'a pas procédé à une collecte et une évaluation systématiques des preuves. Au lieu de cela, les preuves recueillies et évaluées qui ont servi à la rédaction de la déclaration de 2005 (11) ont été mises à jour après avoir effectué des recherches bibliographiques au moyen de PubMed. Les descripteurs étaient « insectifuge » ou « moustiquaire » ou " protection individuelle » seuls ou en combinaison avec «voyageur». Les recherches se limitaient aux articles publiés depuis 2005. Les auteurs ont également parcouru les publications d'entomologie, d'abord en consignant les mêmes descripteurs dans le système de recherche documentaire du Armed Forces Pest Management Board (12) et, dans un deuxième temps, en recensant d'autres données publiées et inédites, notamment des entretiens avec des organismes nationaux de réglementation des pesticides. Des critères d'inclusion spécifiques n'ont pas été utilisés; la pertinence était plutôt attribuée en fonction de la nouveauté (p. ex., une nouvelle approche disponible depuis 2005) et la robustesse (p. ex., des essais aléatoires ou des paramètres pertinents aux voyageurs).

- Le CCMTMV admet que des études bien exécutées sur un échantillon aléatoire de voyageurs fourniraient des preuves de qualité pour les MPI et que ces études font défaut. II y a toutefois eu un certain nombre d'études sur échantillon aléatoire réalisées au sujet des MPI (surtout les moustiquaires de lit) auprès de populations locales de zones d'endémicité. S'il est certain que l'extrapolation de ces données aux voyageurs diminue la qualité de ces mêmes données (13), le CCMTMV estime qu'elles peuvent tout de même justifier la formulation de recommandations rigoureuses, particulièrement lorsque des paramètres pertinents ( $p$. ex., la prévention de la phase clinique d'une maladie) et des populations pertinentes (p. ex., tous âges) sont inclus ou que l'effet est important, étendu et homogène (14).

- Le CCMTMV est convaincu que les mécanismes de réglementation des pesticides axée sur les risques au Canada comme aux États-Unis sont fiables et prudents $(15 ; 16)$. Ceci témoigne de l'importance qu'accordent les organismes de réglementation à l'innocuité des produits en appliquant des facteurs d'innocuité multiples et multiplicatifs. Par conséquent, les produits de protection individuelle approuvés par ces mécanismes sont considérés comme ayant d'excellents profils en ce qui a trait à leur innocuité et ne devraient pas présenter de risques relevant des décisions prises en matière de santé.

- Le CCMTMV constate que bon nombre de preuves concernant les insectifuges proviennent d'études entomologiques relativement restreintes qui s'intéressaient davantage à la protection contre les piqûres et les morsures qu'à la prévention de maladies. De l'avis du CCMTMV, prises individuellement, les études ne fournissent pas de preuves de qualité quant à la prévention des maladies. II considère toutefois que la protection contre les piqûres et les morsures est indicateur raisonnable, quoiqu'indirect, de la prévention de la maladie clinique. Par conséquent, lorsqu'il y a une constance dans les résultats d'une étude à l'autre et que l'effet escompté est ample, le CCMTMV considère que le résultat de l'ensemble des études suffit à justifier une recommandation sur l'emploi des produits.

- Le CCMTMV est conscient qu'il existe une multitude de combinaisons de vecteurs et de maladies et que la plupart de ces vecteurs n'ont pas encore fait l'objet de recherches en profondeur au sujet des MPI qui 
permettraient de s'en protéger. Au lieu de cela, l'accent a été porté essentiellement sur l'analyse de la transmission des maladies par les moustiques et des MPI et, par conséquent, sur les preuves les concernant. Quant aux autres vecteurs (p. ex., tiques, mouches tsé-tsé et phlébotomes), il y a moins de preuves, mais ce qui est disponible indique généralement que les MPI qui sont efficaces contre les moustiques le sont aussi contre ces groupes. Par conséquent, les présentes recommandations s'appuient sur les preuves relatives aux moustiques, mais sont considérées comme généralement applicables aux arthropodes vecteurs. Une évaluation systématique de I'utilité des MPI appliquées à chacun des vecteurs n'a pas été réalisée.

- La perspective adoptée par le CCMTMV dans le cadre de I'analyse est celle du voyageur. En général, cela simplifie les choses puisqu'on ne tient pas compte des effets indirects et à plus long terme des MPI sur les populations humaines et celles des vecteurs à l'échelle des collectivités. D'autre part, cela peut amplifier les préoccupations associées à la variabilité en fonction des vecteurs. Par exemple, la résistance de certains vecteurs du paludisme aux insecticides pyréthrinoïdes avec lesquels on imprègne les moustiquaires des lits a été documentée dans certaines régions d'Afrique, bien que l'impact que cette résistance aura à l'échelle des collectivités reste à élucider $(17 ; 18)$. Cependant, comme la résistance aux insecticides semble associée à une probabilité accrue que les moustiques piqueront «à travers " une moustiquaire imprégnée, elle diminuera probablement l'utilité de la moustiquaire de lit à l'échelle individuelle (y compris chez le voyageur).

- L'annexe 3 présente un sommaire des recommandations formulées dans la déclaration.

\section{Recommandations relatives à la protection individuelle}

Si les insectes ne piquent pas, ils ne transmettent pas de pathogènes. Par conséquent, on peut prévenir des MAA en diminuant l'exposition aux arthropodes, que ce soit en empêchant ou en diminuant l'accès à la peau au moyen d'obstacles. Ces tactiques sont complémentaires et l'on devrait les appliquer concurremment. II ne faut pas oublier que les MPI se veulent des instruments permettant d'augmenter la protection et ne remplacent pas la

\section{Éviter les arthropodes}

Il existe plusieurs façons d'éviter les arthropodes et il va de soi qu'elles pourraient atténuer le risque de contracter une MAA. Cependant, il y a peu de données scientifiques de première main pour soutenir cette hypothèse. Malgré ces lacunes, le CCMTMV estime que les mesures de prévention qui suivent devraient être utiles et que, dans la mesure du possible, elles devraient être appliquées puisqu'il est peu probable qu'elles comportent un risque.

1. Éviter de voyager dans des régions à risque au cours des saisons où la probabilité d'une transmission de MAA est plus élevée.

2. Diminuer l'exposition en évitant les périodes et les lieux où l'on sait que les vecteurs sont actifs (p. ex., en restant à l'intérieur pendant les périodes de forte activité, en diminuant l'exposition dans des zones rurales ou d'autres milieux associés à des vecteurs précis). vaccination ni la prise de médicaments indiquées (p. ex., la chimioprophylaxie pour prévenir le paludisme) (19).

De toute évidence, ce point présuppose que des médicaments ou des vaccins contre les MAA existent, ce qui n'est pas le cas de certaines (comme la dengue et la leishmaniose), c.-à-d. que les MPI sont souvent la seule tactique permettant d'atténuer le risque de contagion.

\section{BARRIÈRES PHYSIQUES}

Il y a une panoplie de barrières physiques qui peuvent être utilisées pour diminuer le contact entre vecteurs et leurs hôtes humains. Certains s'appliquent facilement au moment de l'exposition (p. ex., le port de vêtements appropriés, l'emploi de tulles pour moustiquaire), alors que d'autres, comme les moustiquaires aux fenêtres et aux portes, la climatisation et le cloisonnement des avant-toits exigent parfois d'y penser à l'avance pour s'assurer que ces barrières sont en place. Voici les recommandations qu'émet le CCMTMV au sujet de l'emploi de barrières physiques :

\section{Protéger les lieux de travail et d'hébergement}

contre les vecteurs Ceci peut se faire en installant des moustiquaires aux portes et aux fenêtres, en cloisonnant les avant-toits, en bouchant les trous des toits et des murs et en cloisonnant les espaces autour d'un bâtiment. Ces interventions ont été liées par le passé à des diminutions du risque de paludisme en Amérique du Nord et en Europe $(20 ; 21)$ et ont été associées à 
la diminution du nombre de moustiques vecteurs du paludisme qui entrent dans les huttes et des cas d'anémie chez les occupants de ces huttes, dans une récente étude à échantillon aléatoire en Afrique recourant à l'analyse du projet thérapeutique (22). Plusieurs études à petite échelle en Afrique ont aussi démontré qu'en " grillageant » les habitations, y compris en cloisonnant les avant-toits, il est possible de diminuer significativement le nombre de moustiques vecteurs du paludisme qui s'infiltrent dans les bâtiments (23).

2. Utiliser des moustiquaires de lit Le CCMTMV recommande l'emploi de moustiquaires de lit imprégnées d'un insecticide (MII), bien qu'il y ait des preuves démontrant que des moustiquaires non traités fournissent une protection significative contre les piqûres et les morsures de moustiques et d'autres arthropodes. On estime que les moustiquaires de lit, traitées et non traitées, agissent aussi comme barrière contre $d$ 'autres bestioles (p. ex., les punaises, les rongeurs et les scorpions); certaines données suggèrent qu'elles peuvent diminuer le risque de morsures de serpents venimeux (24).

3. Porter des vêtements appropriés Certains ouvrages en entomologie démontrent qu'en recouvrant la peau avec un tissu, on peut diminuer les piqûres de moustiques (25), bien que cela dépende du genre de tissu, du nombre de couches de tissus et de l'armure. Certaines données suggèrent par ailleurs que les vêtements peuvent protéger contre le paludisme $(26 ; 27)$, mais la qualité de ces données est contestable. Malgré des preuves aussi limitées, le CCMTMV recommande de porter des vêtements appropriés (pleine longueur, amples, de couleur claire) et de les porter correctement (manches déroulées, extrémités du pantalon enfilées dans les chaussettes, les chaussures ou les bottes) afin de se protéger contre les piqûres et les morsures des arthropodes. Cette opinion est justifiée par la prise en compte de trois facteurs à la fois : d'abord, certaines preuves fondées sur les observations entomologiques vont dans le sens de cette intervention et, d'un point de vue mécaniste, cela est plausible; deuxièmement, il est peu probable que le port de vêtements appropriés présente un risque qui remettrait en cause la prise de décisions en matière de santé; troisièmement, le port de ces vêtements a d'autres avantages, comme la protection contre les rayons du soleil.

Fait intéressant, les vêtements de couleurs claires permettent à celui qui les porte de repérer les tiques et de les enlever avant qu'elles ne mordent $(28 ; 29)$.

\section{BARRIÈRES CHIMIQUES}

Bien que ce soit plutôt arbitraire, nous établissons une distinction entre deux types de barrières chimiques : les insectifuges, habituellement topiques, dont le principal effet est d'empêcher les insectes et autres arthropodes de piquer ou de mordre, et les insecticides, qui généralement tuent les insectes et autres arthropodes dès qu'il y a contact ou peu après. En réalité, les activités peuvent se chevaucher puisque des insecticides agissent parfois comme insectifuges (p. ex., la perméthrine) et certains insectifuges topiques comportent des propriétés intoxicantes (p. ex., le DEET).

\section{Insectifuges topiques}

L'application d'insectifuges topiques sur les surfaces exposées de la peau empêche les arthropodes de piquer ou de mordre, mais ne les empêche pas nécessairement de s'approcher de leur hôte. Bien que les insectifuges aient été utilisés depuis des millénaires $(6 ; 7)$, ce n'est surtout que depuis un siècle qu'ils font l'objet d'évaluations et de développements reposant sur la science. Ajoutons que ce n'est que tout récemment que l'on comprend un peu mieux le mode d'action du DEET et de ses analogues (30-32); il semble que ces composés ont un effet direct sur les sensilles des insectes. Parallèlement à ces efforts, il y a eu une véritable explosion de recherches sur de nouveaux produits insectifuges, notamment sur les composés « naturels » (7). Il ne faut pas s'étonner de l'existence d'insectifuges d'origine naturelle étant donné la multitude de produits chimiques provenant de végétaux qui ciblent les insectes. En effet, on a posé comme postulat que la sensibilité des arthropodes hématophages à certains composés chimiques s'explique par un reliquat de l'évolution de leurs caractéristiques phytotrophiques (7). Cependant, malgré un certain succès sur le plan de la commercialisation, peu d'insectifuges naturels dûment homologués sont offerts sur le marché nord-américain. En outre, plusieurs auteurs de confiance ont mis en garde contre les dangers d'une généralisation selon laquelle naturel égale innocuité (6; 7), c.-à-d. que, a priori, il n'y a pas de raison qui permette de s'attendre à ce qu'un insectifuge naturel qui n'est pas homologué soit efficace et sans danger. Par conséquent, le CCMTMV ne recommande pas l'application d'insectifuges "naturels » (ou autres) qui ne sont pas homologués pour se protéger des piqûres et morsures de moustiques et autres arthropodes.

L'Agence de réglementation de la lutte antiparasitaire (ARLA) de Santé Canada doit procéder à l'analyse de l'efficacité et de l'innocuité des insectifuges topiques et 
autres (et des barrières insecticide; voir ci-dessous) avant qu'ils ne soient vendus et utilisés au Canada. Aux États-Unis, une obligation analogue s'applique; c'est la Environmental Protection Agency (EPA) qui est responsable de l'homologation des produits. Dans les deux agences, la priorité est mise sur l'innocuité des produits. L'efficacité est également considérée, bien que les paramètres à cet effet (33) visent à satisfaire aux besoins en matière de réglementation et sont, au mieux, vus comme des mesures indirectes dans le contexte de la protection contre les maladies. Néanmoins, les preuves indiquant que les insectifuges préviennent les piqûres et les morsures d'insectes et autres arthropodes sont impressionnantes et généralement constantes; le CCMTMV estime donc qu'il est très probable que la prévention des piqûres et des morsures diminuera l'exposition aux pathogènes associés aux arthropodes. De plus, il existe des preuves directes tirées d'une étude par échantillon aléatoire selon lesquelles l'application d'un insecticide topique peut diminuer l'incidence du paludisme chez les habitants d'un milieu où sévit une endémie (34).

Voici les recommandations qu'émet le CCMTMV au sujet de l'emploi des insectifuges topiques:

1. Appliquer un insectifuge topique sur les surfaces exposées de la peau pour se prémunir contre les piqûres et les morsures d'arthropodes et atténuer le risque de MAA.

2. Cinq ingrédients actifs sont actuellement homologués au Canada : le DEET, l'icaridine (aussi appelée picaridine et KBR 3023), le p-menthane-3,8-diol (PMD), I'huile de soya et la citronnelle. De tous ces ingrédients, c'est le DEET qui a été utilisé le plus longtemps et a toujours fait preuve d'une grande efficacité. Approuvée pour utilisation au Canada en 2012, I'icaridine (1-acide pipéridinecarboxylique 2-(2-hydroxyéthyl)-1méthylpropylester) fait preuve d'une efficacité comparable au DEET, est disponible dans d'autres territoires depuis quelque temps et est recommandé par les Centers for Disease Control and Prevention (CDC) aux États-Unis $(35 ; 36)$ pour la protection contre les piqûres de moustiques vecteurs. Par ailleurs, elle a été évaluée par l'Organisation mondiale de la santé dans le cadre de son programme d'évaluation des pesticides (Pesticide Evaluation Scheme (WHOPES)) (37; 38); I'OMS la recommande comme étant un « safe and effective insect repellent for human use (insectifuge sécuritaire et efficace lorsqu'utilisé chez l'humain) ». Compte tenu de ces caractéristiques, le DEET et I'icaridine sont recommandés comme insectifuges topiques de première ligne pour se protéger des piqûres et des morsures d'arthropodes et se prémunir des MAA. Il est démontré que l'application $d^{\prime}$ insectifuges topiques contenant du PMD prévient le paludisme (34); les CDC aux États-Unis $(35 ; 36)$ les recommandent pour se protéger contre les piqûres de moustiques vecteurs. Cependant, la teneur en PMD des produits actuellement sur le marché au Canada ne dépasse pas les $10 \%$; on estime que le produit n'offre pas la même fenêtre de protection que celle qu'offrent des concentrations plus élevées de DEET et d'icaridine. De plus, l'application d'insectifuges contenant du PMD est contre-indiquée chez les enfants de moins de trois ans. Pour ces motifs, les produits contenant du PMD devraient être considérés comme une deuxième option pour utilisation par les voyageurs canadiens. D'autres ingrédients actifs actuellement homologués comme insectifuges topiques au Canada (p. ex., la citronnelle et I'huile de soya) ne sont pas disponibles partout ou n'offrent pas une protection d'une durée suffisante contre les piqûres et les morsures $(39 ; 40)$; leur utilisation n'est donc pas recommandée pour protéger les voyageurs contre les piqûres et les morsures de vecteurs.

3. En règle générale, plus la concentration de DEET (annexe 4) ou d'icaridine est élevée, plus la période de protection contre les piqûres et les morsures est longue. Actuellement, les concentrations maximales permises au Canada pour l'application chez les adultes (civils) sont de $30 \%$ pour le DEET auquel sont ajoutés des toluamides apparentés comme matière active, ou de $20 \%$ pour l'icaridine. Le CCMTMV estime que les produits dont les concentrations sont plus élevées ont I'avantage de ne pas nécessiter des applications aussi fréquentes et recommande donc que les adultes utilisent des produits contenant au moins $20 \%$ de DEET ou $20 \%$ d'icaridine. Chez les enfants, l'ARLA permet actuellement l'application de DEET à une teneur d'au plus $10 \%$, et ce, à raison de trois fois par jour dans le cas d'enfants âgés de deux à douze ans et d'une fois par jour dans le cas d'enfants âgés de six mois à deux ans. Par contre, il est permis d'appliquer un insectifuge topique d'une teneur en icaridine d'au plus $20 \%$ sur les enfants âgés d'au moins six mois. Par conséquent, l'insectifuge topique contenant de l'icaridine est préféré pour cette catégorie d'âge alors que celui contenant du DEET est un deuxième choix. L'ARLA interdit I'application d'insectifuges contenant du DEET ou de l'icaridine sur les nourrissons de moins de six mois (41) (document de la décision d'homologation de l'icaridine). Le CCMTMV prend acte de cette réglementation, mais signale qu'elle n'a pas été élaborée pour prévenir les MAA, mais plutôt pour tenir compte d'une prise de décisions réglementaires fondée sur le contexte canadien de prévention des 
désagréments causés par les piqûres et les morsures. Conséquemment, le CCMTMV est d'avis que, pour les déplacements à l'extérieur du Canada dans des pays où certaines MAA sont endémiques ou épidémiques et où une exposition est probable, le risque de contracter une MAA est supérieur au risque d'une réaction indésirable au DEET ou à l'icaridine. Dans ces situations et lorsque des vecteurs ne peuvent pas être écartés autrement (p. ex., en utilisant des moustiquaires imprégnées d'insecticide), l'application de DEET ou d'icaridine à une teneur d'au plus $10 \%$ devrait être envisagée pour les nourrissons de moins de six mois. Le produit doit être appliqué selon le mode d'emploi pour les nourrissons > six mois d'âge.

4. Au besoin, répéter l'application de l'insectifuge. Les étiquettes des insectifuges topiques indiquent souvent une estimation du temps de protection, par exemple "protège contre les moustiques pendant six heures ». Bien que ces valeurs s'appuient sur une évaluation des données sur l'efficacité, elles ne sont pas élaborées selon le contexte du risque de contracter une MAA, et ne tiennent pas suffisamment compte des variations qui s'expliquent par d'autres facteurs comme la sueur (42), les espèces de moustiques (43) et le nombre de moustiques (44). Par conséquent, dans le cas de séjours à l'étranger dans des zones où les MAA constituent une menace, il serait raisonnable d'appliquer l'insectifuge plus souvent que ne l'indique l'étiquette. En principe, si l'on remarque des piqûres ou des morsures avant que l'intervalle indiqué sur l'étiquette ne prenne fin, alors une nouvelle application de l'insectifuge est recommandée. Si l'on souhaite diminuer au minimum I'application d'un insectifuge, le traitement peut être chronométré de sorte à optimiser la protection avec un nombre minimal d'applications. Par exemple, en appliquant le matin une formule de protection de longue durée avant ou immédiatement après être sorti d'une moustiquaire de lit traitée et en l'appliquant à nouveau en fin d'après-midi pour la période qui va du souper à l'entrée sous la moustiquaire devrait offrir une protection au cours de la période de haute activité des vecteurs du paludisme.

5. Les voyageurs ne comprennent pas toujours l'importance d'appliquer des MPI (45-47). Bien qu'il y ait peu de données portant sur l'évaluation d'une meilleure conformité des interventions ciblées, il est vraisemblable que les préférences personnelles jouent un rôle important sur l'adhésion. Pour cette raison, le CCMTMV encourage les praticiens et les voyageurs à adapter les recommandations et les choix d'insectifuges selon les préférences personnelles (p. ex., certaines personnes préfèrent une crème insectifuge alors que d'autres préfèrent un aérosol).
6. L'ARLA ne milite pas en faveur de l'utilisation de produits qui conjuguent un insectifuge et un écran solaire; par ailleurs, le CCMTMV ne recommande pas d'utiliser ces produits. Ceci s'explique par les contradictions des modes d'emploi de ces produits : les écrans solaires doivent être appliqués souvent et généreusement alors que les insectifuges le devraient moins et moins souvent. Il y a aussi des preuves qu'une administration simultanée peut diminuer l'efficacité de l'écran solaire et, à l'inverse, augmenter l'absorption de DEET par voie cutanée (48-52), bien qu'il est possible que ce ne soit pas le cas de l'icaridine (50). Comme l'interaction entre un écran solaire et un insectifuge diminuera probablement à mesure que s'écoule l'intervalle entre leurs applications respectives, il est recommandé, s'il faut appliquer les deux, d'appliquer d'abord l'écran solaire et lui permettre de pénètrer la peau (p. ex., pendant 15 minutes) avant d'appliquer l'insectifuge. Lorsqu'il est impossible de procéder ainsi, le CCMTMV estime que l'utilité protectrice de ces interventions l'emporte sur les risques que pourrait poser leur administration simultanée, c.-à-d. que l'application des deux est recommandée même si l'écran solaire ne peut pas être appliqué avant l'insectifuge.

\section{Barrières insecticides}

Une multitude de stratégies de barrières à base d'insecticides peuvent être utilisées pour diminuer l'exposition aux piqûres et morsures de vecteurs. En effet, certaines de ces approches (p. ex., les moustiquaires de lit imprégnées d'un insecticide (MII) et la pulvérisation d'insecticides à effet rémanent à l'intérieur des habitations $(\mathrm{PIERIH)),} \mathrm{représentent} \mathrm{les} \mathrm{principales} \mathrm{interventions} \mathrm{de} \mathrm{lutte}$ antipaludique dans les pays où cette maladie est endémique. Elles offrent une bonne protection contre le paludisme, y compris une diminution significative de la mortalité infantile, toutes causes confondues (53-55). De ces deux interventions, la présente déclaration portera strictement sur l'utilisation de MII puisque la PIERIH est appliquée par des professionnels dans un contexte d'endémicité et échappe donc au contrôle de la plupart des voyageurs. Cependant, pour les voyageurs à long terme, les expatriés, les personnes qui passent beaucoup de temps dans des zones rurales à risque, entre autres, ce ne serait pas déraisonnable de privilégier des logements ou des zones qui font l'objet d'une PIERIH. Pour obtenir de plus amples renseignements sur la PIERIH, le lecteur peut consulter le site Web du WHOPES (37), qui présente de l'information sur les essais sur la PIERIH (56) et une liste mise à jour des produits de PIERIH approuvés (57). II faut souligner que la PIERIH n'est pas considérée comme une solution de rechange aux MII, mais plutôt un complément 
à celles-ci, c.-à-d. que les MIl sont recommandées, qu'il y ait PIERIH ou non. Outre les MII, il existe d'autres stratégies de MPI à base d'insecticides, dont le port de vêtements imprégnés d'un insecticide, qui seront traitées plus en détail dans la présente déclaration.

\section{MOUSTIQUAIRES DE LIT}

1. Bien que peu d'études aient été réalisées spécifiquement sur l'évaluation de l'utilité des MII pour les voyageurs, il existe des données fiables qui démontrent qu'elles protègent les populations des zones où le paludisme est endémique (p. ex., diminution des épisodes de paludisme sans complications de I'ordre de 50 \%) (53). Le CCMTMV estime que ces données suffisent à émettre une forte recommandation en faveur de l'utilisation de MII par tous les voyageurs qui se retrouvent dans un pays étranger où certaines MAA sont endémiques ou épidémiques. Les MII sont également recommandées pour se protéger des bestioles porteuses de pathogènes qui se trouvent à l'intérieur des habitations (p. ex., les réduves) ou qui causent des désagréments (p. ex., les punaises).

2. Bien que les MIl et les produits connexes (moustiquaires portables imbibées d'insecticide) soient habituellement déployés la nuit, il faudrait s'en servir selon les besoins, y compris au moment de se reposer à l'intérieur d'une habitation durant le jour et pour protéger les enfants au berceau ou en poussette.

3. Au Canada, les moustiquaires de lit imbibées d'insecticide sont réglementées par l'ARLA. À l'exception de certains produits à usage militaire, aucun produit connexe aux MII n'est actuellement homologué au Canada et il n'existe pas une politique précise qui permet leur vente au Canada pour usage à l'étranger. Néanmoins, les MIl peuvent s'obtenir auprès de certaines cliniques santé-voyage au Canada et de fournisseurs au pays et à l'étranger. Quoiqu'il serait préférable d'avoir des MII homologuées au Canada, le CCMTMV est d'avis qu'il est peu probable qu'il y aura une homologation de MII dans un proche avenir. Cependant, l'Organisation mondiale de la santé offre, dans le cadre de son programme WHOPES, un processus par lequel il est possible d'évaluer leur innocuité et leur efficacité $(37 ; 58)$. À cet égard, le WHOPES recommande des formules précises $d^{\prime}$ insecticides pour le traitement des MII conventionnels (annexe 5) $(59 ; 60)$ et recommande des produits précis (annexe 6) (61) qui satisfont à ses critères (62) comme moustiquaires imprégnées d'un insecticide à effet rémanent (MàER). Ce dernier représente à lui seul une catégorie de MII qui satisfont à des paramètres très stricts en matière de rendement (p. ex., une fois déployée, l'effet doit durer au moins trois ans, le maintien de l'efficacité après au moins 20 lavages). Le CCMTMV est d'avis que le programme WHOPES est fiable et recommande de sélectionner des moustiquaires de lit en fonction des recommandations provisoires et définitives du WHOPES, qu'il s'agisse de la formule insecticide (59) ou d'un produit précis de MàER (61).

4. Plusieurs MII ou traitements de MII (p. ex., Insect Shield ${ }^{\circledR}$ ), y compris des MàER (c.-à-d. les moustiquaires Pramex ${ }^{\circledR}$ et Duration ${ }^{\circledR}$; homologation aux É.-U. par I'EPA, numéro 73049-433; les moustiquaires sont fabriquées à partir de la technologie Olyset ${ }^{\circledR}$ ) ont été homologués par I'EPA aux États-Unis. Lorsque ces MII sont fabriquées à partir de technologies qui font l'objet d'une recommandation du programme WHOPES (p. ex., Olyset ${ }^{\circledR}$, annexe 6), elles sont préférées aux produits sans homologation de I'EPA aux États-Unis; ou recommandés par WHOPES, mais non homologués par I'EPA. Si I'on devait procéder à l'homologation de MII au Canada, un algorithme analogue s'appliquerait, en accordant la préférence aux produits homologués au Canada par rapport à ceux homologués aux États-Unis.

5. Il y a plusieurs types de MII sur le marché mondial, mais les normes de fabrication ne sont pas toujours les mêmes. Voilà pourquoi le CCMTMV recommande I'utilisation de moustiquaires qui sont homologués au Canada ou aux États-Unis et/ou lorsque le fournisseur ou le fabricant peut présenter les documents attestant leur conformité aux spécifications de WHOPES. Fait important : certaines moustiquaires de lit sont commercialisées en qualité de MàER alors qu'elles ne sont pas désignées ainsi par le WHOPES. Le CCMTMV recommande de ne pas utiliser ces produits en qualité de MàER ou de ne pas les commercialiser sous cette étiquette.

6. Afin de réduire au minimum une exposition aux pesticides qui pourrait se produire lors de déversements ou un mauvais traitement des moustiquaires, le CCMTMV recommande aux voyageurs d'utiliser des moustiquaires prétraitées au lieu de moustiquaires qu'ils ont traités eux-mêmes. Lorsque cela est impossible, il faut appliquer les lignes directrices du WHOPES en matière de traitement et de prétraitement (63).

7. Pour la plupart des voyageurs, les MII conventionnelles devraient offrir une protection suffisamment longue. Cependant, lorsque les déplacements dans des zones à risque sont fréquents et/ou que les séjours prévus sont longs (p. ex., six mois ou plus), les MàER peuvent être avantageuses. 
8. Dans certaines régions, les moustiques vecteurs du paludisme ont acquis une résistance aux insecticides (c.-à-d. aux insecticides pyréthrinoïdes) qui sont actuellement utilisés pour traiter les MII. On ne connaît pas très bien l'impact que cette résistance a ou aura $(17 ; 18)$. À ce stade-ci, si l'on fait abstraction de l'emploi des techniques supplémentaires et complémentaires déjà mentionnées dans la présente déclaration, le CCMTMV n'a pas de recommandations particulières au sujet de l'utilisation de matériaux traités avec un insecticide lorsque la résistance a été documentée.

\section{TRAITEMENTS DES VÊTEMENTS}

9. Il est démontré que le port de vêtements imprégnés d'un insecticide (VII) protège des piqûres et des morsures d'arthropodes (de 64 à 67) et qu'il peut également protéger des MAA (68). Le port de VII est donc recommandé pour se prémunir contre les MAA. Les produits pour VII actuellement homologués au Canada contiennent de la perméthrine et servent à des fins militaires seulement. Aux États-Unis toutefois, plusieurs de ces produits pour VII contenant de la perméthrine sont sur le marché et servent à des fins civiles. Ils sont généralement disponibles sous forme d'apprêt contenant 0,5\% de perméthrine et que le consommateur applique lui-même sur son vêtement ou un vêtement prétraité avec de la perméthrine à teneur de 0,52\%, masse pour masse. Ces approches se distinguent en ce que la dernière approche, d'après l'étiquette, fournit une protection après des dizaines de lavages alors que le produit à $0,5 \%$ protège encore après plusieurs lavages. Les deux sont disponibles auprès des grands détaillants en ligne aux États-Unis; les consommateurs canadiens devraient pouvoir les obtenir pour un usage personnel ${ }^{1}$ (69). Pour tous les vêtements traités ou le traitement des vêtements, I'utilisateur doit suivre les consignes mentionnées sur l'étiquette. Quant aux insectifuges topiques et aux MII, jusqu'au moment où les VII seront homologués au Canada, ce sont les produits homologués par l'EPA aux États-Unis qui sont préférables à ceux disponibles à l'étranger.

10. Afin d'optimiser la protection contre les piqûres et les morsures, il faut porter des VII avec le produit insectifuge adapté aux circonstances (66; 70), de préférence à effet rémanent, de sorte que la peau soit presque totalement protégée (c.-à-d. qu'il faut appliquer un insectifuge topique sur la peau exposée parce que non recouverte par un VII).
11. Dans le cas de certains vecteurs, les VII sont considérés relativement plus importants. Par exemple, I'on estime que les VII procurent une excellente protection contre les morsures de tiques qui transmettent la Borrelia burgdorferi, l'agent responsable de la maladie de Lyme (67). D’autres données encore semblent indiquer que, pendant la Seconde Guerre mondiale, les militaires dont les vêtements étaient traités ont été épargnés du typhus des broussailles (71).

\section{AUTRES PRODUITS INSECTICIDES}

12. Il existe une multitude d'autres approches basées sur les insecticides qui prétendent contrer les arthropodes vecteurs et/ou les désagréments comme les punaises de lit. Ils comprennent les spirales d'insecticides qui se brûlent, les insecticides en atomiseur, en aérosol et en pulvérisations spatiales, ainsi que les draps traités avec un insecticide. Plusieurs de ces interventions se sont avérées décisives lors des campagnes contre le paludisme tout au long de I'histoire. Par exemple, la fumigation des habitations au moyen de divers produits a d'abord été appliquée dans le cadre d'une stratégie intégrée en vue de contrôler et de contrer la prolifération des vecteurs du paludisme au cours de la construction du canal de Panama et de permettre au Brésil de venir à bout du vecteur du paludisme venu d'Afrique, I'A. gambiae (72; 73). Cependant, certaines de ces approches se traduisent par la dispersion de quantités relativement importantes $d^{\prime}$ insecticides à I'intérieur des bâtiments et ne satisfont probablement plus aux normes en matière d'innocuité qui sont en vigueur de nos jours. D'autres, par exemple les vaporisateurs thermiques maintenant approuvés au Canada et aux États-Unis, se butent à un manque de données qui démontreraient leur efficacité sur le plan de la santé publique. Enfin, alors qu'ils sont prétendument efficaces contre les punaises et peut-être même contre les vecteurs du paludisme (74), certains produits comme les draps traités sont probablement associés à une exposition plus importante aux insecticides en raison d'un contact accru entre la peau et l'objet traité.

Le CCMTMV n'a pas effectué une revue de la littérature approffondie sur ces autres approches. Par conséquent, le degré de confiance qu'il attribue à son évaluation n'est pas élevé, mais cela ne l'empêche pas de recommander de ne pas appliquer les approches susmentionnées aux

\footnotetext{
Règlement sur les produits antiparasitaires. Alinéa 3(1)f) « Les produits antiparasitaires ci-après sont exemptés de l'application de la Loi : [...] le produit antiparasitaire - sauf un organisme - qui est importé au Canada principalement pour l'usage de l'importateur dans des lieux d'habitation ou autour de ceux-ci, dont la quantité n'excède pas $500 \mathrm{~g}$ ou $500 \mathrm{~mL}$ et dont la valeur ne dépasse pas 100 \$. » (69).
} 
fins de prévention des MAA ou des désagréments causés par les piqûres et les morsures. Cette décision se fonde sur la juxtaposition de plusieurs facteurs : l'incertitude au sujet du profil de l'innocuité de certaines de ces interventions,

\section{MPI inefficaces}

Plusieurs produits sur le marché sont commercialisés comme étant des MPI efficaces. Dans certains cas, il n'y a toutefois pas suffisamment de preuves scientifiques permettant de confirmer les allégations sur leur efficacité ou leur innocuité. Citons en exemple les dispositifs électroniques (à ultrasons) $(75 ; 76)$, les bracelets, colliers et bandes aux chevilles imprégnés d'insectifuge (40), les dispositifs d'électrocution $(75 ; 77)$, les pièges qui attirent
I'incertitude au sujet de leur efficacité ou la disponibilité de mesures de rechange qui ont des profils d'efficacité démontrée et d'innocuité acceptable (p. ex., les MII, les VII et les insectifuges topiques).

les moustiques par l'odeur (de 78 à 80$)$, le citrosa $(78 ; 81 ; 82)$, la vitamine B1 administrée par voie orale (83) et les hydratants pour la peau qui ne renferment pas un ingrédient actif à action insectifuge approuvé (40). Le CCMTMV recommande de ne pas recourir à ces interventions pour se prémunir des piqûres et des morsures d'arthropodes et des MAA qui leur sont associées. 


\section{ANNEXE 1. Information sommaire sur les principaux arthropodes vecteurs (de 84 à 88 )}

\begin{tabular}{|c|c|c|}
\hline Vecteur & Maladie causée & $\begin{array}{l}\text { Périodes de pointe et endroits où se } \\
\text { produisent les piqûres ou les morsures }\end{array}$ \\
\hline Moustiques du genre Anopheles & $\begin{array}{l}\text { Filariose } \\
\text { Paludisme }\end{array}$ & $\begin{array}{l}\text { Piquent habituellement entre le crépuscule } \\
\text { et l'aurore. Les heures de pointe pour les } \\
\text { piqûres peuvent varier. Certaines espèces } \\
\text { préfèrent piquer à l'intérieur, alors que } \\
\text { d'autres préfèrent l'extérieur. }\end{array}$ \\
\hline Moustiques du genre Aedes & $\begin{array}{l}\text { Dengue } \\
\text { Filariose } \\
\text { Fièvre jaune }\end{array}$ & $\begin{array}{l}\text { Peuvent piquer à toute heure de la journée, } \\
\text { souvent à l'extérieur, mais certains vecteurs } \\
\text { importants piquent à l'intérieur. }\end{array}$ \\
\hline Moustiques du genre Culex & $\begin{array}{l}\text { Filariose } \\
\text { Encéphalite japonaise } \\
\text { Virus du Nil occidental }\end{array}$ & $\begin{array}{l}\text { Piquent habituellement entre le crépuscule } \\
\text { et l'aurore, souvent à l'extérieur, mais parfois } \\
\text { à l'intérieur. }\end{array}$ \\
\hline Moustiques du genre Mansonia & Filariose & $\begin{array}{l}\text { Piquent habituellement la nuit, surtout } \\
\text { à l'extérieur. }\end{array}$ \\
\hline Moucherons & $\begin{array}{l}\text { Mansonellose } \\
\text { Fièvre à virus Oropouche }\end{array}$ & $\begin{array}{l}\text { Peuvent piquer à toute heure de la journée, } \\
\text { habituellement à l'extérieur. }\end{array}$ \\
\hline Tiques & $\begin{array}{l}\text { Anaplasmose } \\
\text { Babésiose } \\
\text { Fièvre hémorragique de Congo-Crimée } \\
\text { Ehrlichiose } \\
\text { Maladie de Lyme } \\
\text { Encéphalite à virus Powassan } \\
\text { Fièvre } Q \\
\text { Fièvre pourprée des montagnes Rocheuses } \\
\text { Encéphalite à tiques } \\
\text { Fièvre récurrente à tiques }\end{array}$ & $\begin{array}{l}\text { Très dispersées dans les forêts, les prés et } \\
\text { les zones herbeuses; attendent sur un brin } \\
\text { d'herbe ou la branche d'un buisson bas qu'un } \\
\text { hôte passe. Certaines espèces sont plus } \\
\text { agressives et cherchent activement un hôte. }\end{array}$ \\
\hline Puces & $\begin{array}{l}\text { Typhus murin } \\
\text { Peste } \\
\text { Tularémie }\end{array}$ & $\begin{array}{l}\text { Présentes à l'intérieur et à l'extérieur, souvent } \\
\text { associées à un type d'hôte précis, mais se } \\
\text { nourriront sur divers mammifères, y compris } \\
\text { les humains. }\end{array}$ \\
\hline Poux de corps & $\begin{array}{l}\text { Typhus exanthématique } \\
\text { Fièvre récurrente à poux } \\
\text { Fièvre des tranchées }\end{array}$ & $\begin{array}{l}\text { Présents habituellement dans les } \\
\text { vêtements sauf lorsqu'ils se nourrissent } \\
\text { sur l'hôte humain. }\end{array}$ \\
\hline Phlébotomes & $\begin{array}{l}\text { Leishmaniose } \\
\text { Fièvre à phlébotome }\end{array}$ & $\begin{array}{l}\text { Piquent habituellement la nuit; un grand } \\
\text { nombre piquent à l'extérieur, mais certains } \\
\text { s'alimentent également à l'intérieur. }\end{array}$ \\
\hline Mouches noires & Onchocercose chez l'humain & $\begin{array}{l}\text { Piquent habituellement le jour, à l'extérieur, } \\
\text { près des rivières ou des ruisseaux. }\end{array}$ \\
\hline Mouches à chevreuil et à cheval (tabanidés) & $\begin{array}{l}\text { Loase } \\
\text { Tularémie }\end{array}$ & $\begin{array}{l}\text { Actives le jour, à l'extérieur, souvent au cours } \\
\text { des heures les plus ensoleillées; s'alimentent } \\
\text { rarement à l'intérieur. }\end{array}$ \\
\hline Mouches tsé-tsé (glossines) & Trypanosomiase africaine & $\begin{array}{l}\text { Actives habituellement durant le jour (mais } \\
\text { certaines espèces vectrices importantes sont } \\
\text { actives au crépuscule ou à l'aube) et piquent } \\
\text { habituellement à l'extérieur. }\end{array}$ \\
\hline Triatomes ou réduves & Trypanosomiase américaine & $\begin{array}{l}\text { Actifs la nuit et se nourrissent de sang } \\
\text { humain, habituellement à l'intérieur. }\end{array}$ \\
\hline
\end{tabular}

REMARQUE : les références (87 et 88), en anglais et en français respectivement, présentent une série de tableaux détaillés sur les vecteurs, les maladies auxquelles ils sont associés et les régions à risque. 


\section{ANNEXE 2. Force et qualité des preuves (10)}

\begin{tabular}{l|l}
\hline \multicolumn{2}{l}{ Catégories relatives à la fermeté de chaque recommandation } \\
\hline Catégorie & Définition \\
\hline A & Preuves suffisantes pour recommander l'utilisation. \\
\hline B & Preuves acceptables pour recommander l'utilisation. \\
\hline C & Preuves insuffisantes pour recommander ou déconseiller l'utilisation. \\
\hline D & Preuves acceptables pour déconseiller l'utilisation. \\
\hline E & Preuves suffisantes pour déconseiller l'utilisation. \\
\hline
\end{tabular}

\begin{tabular}{l|l}
\hline \multicolumn{2}{l}{ Catégories relatives à la qualité des preuves sur lesquelles les recommandations } \\
\hline Catégorie & Définition \\
\hline I & Données obtenues dans le cadre d'au moins un essai comparatif convenablement randomisé. \\
\hline II & $\begin{array}{l}\text { Données obtenues dans le cadre d'au moins un essai clinique bien conçu, sans randomisation, d'études de cohortes } \\
\text { ou d'études analytiques cas-témoins, réalisées de préférence dans plus d'un centre, à partir de plusieurs séries } \\
\text { chronologiques, ou résultats spectaculaires d'expériences non comparatives. }\end{array}$ \\
\hline III & $\begin{array}{l}\text { Opinions exprimées par des sommités dans le domaine et reposant sur l'expérience clinique, des études descriptives } \\
\text { ou des rapports de comités d'experts. }\end{array}$ \\
\hline
\end{tabular}

\section{ANNEXE 3. Recommandations dans le cadre d'une approche de la médecine fondée sur les preuves (MFP)}

\section{Recommendations}

CatégorieMFP (10)

\section{Éviter les arthropodes}

1. Éviter de voyager dans des régions à risque au cours des saisons où la probabilité d'une transmission de MAA est plus élevée.

2. Diminuer l'exposition en évitant les périodes et les lieux où l'on sait que les vecteurs sont actifs (p. ex., en restant à l'intérieur pendant les périodes de forte activité, en diminuant l'exposition dans des zones rurales ou d'autres milieux associés à des vecteurs précis).

\section{Barrières physiques}

1. Installer des moustiquaires aux portes et aux fenêtres, cloisonner les avant-toits, boucher les trous des toits et des murs et fermer les autres espaces permettant ainsi de protéger les lieux de travail et d'hébergement contre les vecteurs.

2. Porter des vêtements appropriés (p. ex., des vêtements pleine longueur, amples, de couleurs claires).

1. Appliquer sur les surfaces exposées de la peau un insectifuge topique homologué au Canada afin de se prémunir contre les piqûres et les morsures d'arthropodes et atténuer le risque de MAA.

i. Des insectifuges contenant du DEET (de 20 à $30 \%$ ) ou de l'icaridine (20\%) devraient être le premier choix pour les adultes.

ii. Des insectifuges contenant de l'icaridine (20\%) devraient être le premier choix pour les enfants âgés de six mois à douze ans. Des insectifuges contenant des concentrations de DEET en fonction de l'âge devraient être considérés comme une deuxième option pour les enfants âgés de six mois à douze ans.

iii. Les insectifuges contenant du p-menthane-3,8-diol devraient être considérés comme une deuxième option d'insectifuges topiques destinés aux adultes et aux enfants de plus de trois ans.

iv. D'autres ingrédients actifs actuellement homologués au Canada (p. ex., la citronnelle et l'huile de soya) ne sont pas disponible partout ou n'offrent pas une protection d'une durée suffisante contre les piqûres et les morsures; leur utilisation n'est donc pas recommandée pour protéger les voyageurs contre les piqûres et les morsures de vecteurs.

v. Dans le cas de déplacements à l'étranger vers des régions endémiques ou épidémiques, le risque de contracter des maladies associées aux arthropodes (MAA) est supérieur au risque d'une réaction indésirable au DEET ou à l'icaridine. Dans ces situations et lorsque des vecteurs ne peuvent pas être écartés autrement (p. ex., en utilisant des moustiquaires imprégnées d'insecticide), l'application de DEET à une teneur maximale de $10 \%$ ou d'icaridine à une teneur de $10 \%$ devrait être envisagée pour les nourrissons de moins de six mois. 
Recommendations

CatégorieMFP (10)

vi. Si l'on remarque des piqûres ou des morsures avant que l'intervalle indiqué sur l'étiquette pour une nouvelle application n'ait pris fin, il faut appliquer l'insectifuge de nouveau.

vii. Lorsqu'on a intérêt à diminuer la quantité d'insectifuge à utiliser, il faut l'appliquer lorsque les vecteurs sont le plus actifs et où l'exposition à ces vecteurs est plus probable (p. ex., en se levant du lit doté d'une moustiquaire le matin et tard l'après midi).

viii. Dans la mesure du possible, opter en faveur de MPI qui sont adaptées aux besoins de chaque voyageur (p. ex., un insectifuge topique sous forme de liquide, de crème ou en atomiseur afin d'augmenter la probabilité qu'il soit utilisé).

ix. Ne pas utiliser de produits qui sont à la fois un insectifuge et écran solaire.

x. Il est préférable d'appliquer d'abord un écran solaire et, une fois qu'il a pénétré la peau, d'appliquer un insectifuge. Lorsque ce n'est pas possible, appliquer les deux produits, quitte à le faire en même temps.

\section{Barrières insecticides}

1. Pour les voyageurs à long terme, les expatriés, les personnes qui passent beaucoup de temps dans des zones rurales à risque, entre autres, ce ne serait pas déraisonnable de privilégier les logements ou les endroits dont les intérieurs font l'objet d'une pulvérisation à effet rémanent.

\section{Moustiquaires de lit}

1. Utiliser des moustiquaires imprégnées d'un insecticide à effet rémanent (MàER) pour se prémunir des piqûres et des morsures d'arthropodes et des maladies qui leur sont associées.

i. Bien que les MII et les produits connexes (moustiquaires portables imbibées d'insecticide) soient habituellement déployés la nuit, il faudrait s'en servir selon les besoins, y compris au moment de se reposer le jour à l'intérieur d'une habitation et pour protéger les enfants dans les berceaux ou les poussettes.

ii. Dans la mesure du possible, il faut sélectionner les moustiquaires de lit en fonction de leur homologation par I'ARLA ou l'EPA et d'une recommandation provisoire ou définitive de l'Organisation mondiale de la santé dans le cadre de son programme Pesticide Evaluation Scheme (WHOPES).

iii. Utiliser des produits qui sont fabriqués là où l'assurance de la qualité est plus élevée (p. ex., de pays occidentaux ou de fournisseurs et fabricants qui sont en mesure de présenter des documents attestant leur conformité aux spécifications de WHOPES).

iv. Certains produits sont commercialisés en qualité de MàER alors qu'ils ne sont pas désignés ainsi par le WHOPES. Le CCMTMV n'est pas en mesure d'évaluer la véracité de ces allégations et, par conséquent, recommande de ne pas utiliser ces produits ou de ne pas les commercialiser en qualité de MII à effet rémanent (MàER).

v. Afin de réduire au minimum une exposition aux pesticides qui pourrait se produire lors de déversements ou d'un traitement inadéquat des moustiquaires, utiliser des moustiquaires prétraitées au lieu de moustiquaires qui ont été traitées de façon improvisée.

vi. Lorsqu'on prévoit voyager fréquemment ou pendant longtemps (p. ex., six mois ou plus), utiliser un MàER.

vii. Utiliser une MII pour diminuer les piqûres et les morsures d'arthropodes et se prémunir contre les morsures de serpent.

\section{Traitements des vêtements}

1. Porter des vêtements imprégnés d'un insecticide (VII) afin de se protéger des piqûres et des morsures d'arthropodes vecteurs et d'arthropodes nuisibles.

i. Afin d'optimiser la protection contre les piqûres et les morsures, il faut porter des VII avec un produit insectifuge adapté aux circonstances, de préférence à effet rémanent, de sorte que la peau soit presque totalement protégée, c.-à-d. qu'il faut appliquer un insectifuge topique sur la peau exposée parce que non recouverte par un VII.

ii. Jusqu'au moment où des VII sont homologués à des fins civiles au Canada, porter des VII homologués par la Environmental Protection Agency des États-Unis (US EPA).

B II

C III

C III

E II

CIII

C III

A I

Al

A II

C III

D III

D III

C III

B II

B II

A II

A II 


\section{Autres produits insecticides}

1. Le CCMTMV recommande de ne pas recourir, et de ne pas se fier, à d'autres approches reposant sur des insecticides comme les spirales d'insecticides qui se brûlent, les insecticides en atomiseur, en aérosol et en pulvérisations spatiales, ainsi que les draps traités avec un insecticide.

2. Le CCMTMV recommande de ne pas appliquer de mesures de protection individuelle qui ne sont pas efficaces ou dont l'efficacité contre les arthropodes vecteurs et les maladies qui leur sont associées n'a pas été démontrée de façon convaincante. Parmi celles-ci, notons :

- Les dispositifs électroniques (à ultrasons)

- Les bracelets, colliers et bandes aux chevilles imprégnés d'un insectifuge

- Les dispositifs à électrocution (« bug zappers »)

- Les pièges qui attirent les moustiques par l'odeur

- Le citrosa (plante ornementale de la famille des géraniums)

- Vitamine $B_{1}$ administrée par voie orale

- Hydratants pour la peau qui ne renferment pas un ingrédient actif à action insectifuge approuvé.

ANNEXE 4. Estimation du temps de protection des diverses concentrations de DEET

\begin{tabular}{|c|c|c|c|c|c|c|}
\hline \multirow{3}{*}{ \% DEET } & \multicolumn{6}{|c|}{ Durée de protection complète } \\
\hline & \multicolumn{2}{|c|}{ Moyenne } & \multicolumn{2}{|c|}{ Limite inférieure } & \multicolumn{2}{|c|}{ Limite supérieure } \\
\hline & Minutes & Heures* & Minutes & Heures* & Minutes & Heures* \\
\hline 5 & 110 & 2 & 76 & 1,5 & 144 & 2,5 \\
\hline 10 & 214 & 3,5 & 158 & 2,5 & 270 & 4,5 \\
\hline 15 & 276 & 5 & 208 & 3,5 & 344 & 5,5 \\
\hline 20 & 319 & 5,5 & 243 & 4 & 395 & 6,5 \\
\hline 25 & 352 & 6 & 269 & 4,5 & 469 & 8 \\
\hline 30 & 380 & 6,5 & 281 & 5 & 469 & 8 \\
\hline
\end{tabular}

*Arrondi à la demi-heure la plus près

SOURCE : Santé Canada, Agence de réglementation de la lutte antiparasitaire, RRD 2002-01 (41).

\section{ANNEXE 5. Produits insecticides recommandés par l'OMS pour le traitement des moustiquaires pour se prémunir des vecteurs du paludisme}

1. Traitement conventionnel :

\begin{tabular}{l|l|l}
\hline Insecticide & Formulation $^{1}$ & Dosage $^{\mathbf{2}}$ \\
\hline L'alpha-cyperméthrine & SC 10\% & $20-40$ \\
\hline Cyfluthrine & EW 5\% & 50 \\
\hline Deltaméthrine & SC 1\%; WT 25\%; and WT 25\% + liant ${ }^{3}$ & $15-25$ \\
\hline Étofenprox & EW 10\% & 200 \\
\hline Lambda-cyhalothrine & CS 2,5\% & $10-15$ \\
\hline Perméthrine & EC $10 \%$ & $200-500$ \\
\hline
\end{tabular}


2. Traitement de longue durée :

\begin{tabular}{l|l|l}
\hline Le nom du produit & Type de produit & Statut de la recommendation de I'OMS \\
\hline ICON ${ }^{\circledR} M A X X$ & $\begin{array}{l}\text { Lambda-cyhalothrin } 10 \% \mathrm{CS}+\text { liant } \\
\text { Dose cible de } 50 \mathrm{mg} / \mathrm{m}^{2}\end{array}$ & Intérimaire \\
\hline
\end{tabular}

1 EC = concentrés émulsionnables; EW = émulsions - huile dans l'eau; CS = suspension de capsules; SC - concentrés fluidifiables;

$\mathrm{WT}=$ tablettes dispersables dans l'eau

2 Milligrammes d'ingrédient actif par mètre carré de filet

$3 \mathrm{~K}-\mathrm{O}$ TAB $1-2-3^{\circledR}$

REMARQUE : (1) Les recommandations de I'OMS sur l'utilisation des pesticides en santé publique sont valides SEULEMENT lorsqu'elles sont liées aux spécifications de contrôle de la qualité de l'OMS. Les spécifications de l'OMS pour les pesticides en santé publique sont disponibles sur la page d'accueil de l'OMS sur l'Internet à: www.who.int/whopes/quality/en (2) Pour une liste à jour, consulter le site Web de WHOPES (www.who.int/whopes).

SOURCE : Programme Pesticide Evaluation Scheme (WHOPES) de I'Organisation mondiale de la santé (59).

ANNEXE 6. Moustiquaires imprégnées d'un insecticide à effet rémanent (MIlàER) recommandées par l'OMS

\begin{tabular}{|c|c|c|c|}
\hline $\begin{array}{l}\text { Le nom } \\
\text { du produit }\end{array}$ & Type de produit & $\begin{array}{l}\text { Statut de la } \\
\text { recommendation } \\
\text { de l'OMS }\end{array}$ & $\begin{array}{l}\text { Statut de la publication } \\
\text { des spécifications } \\
\text { de l'OMS }\end{array}$ \\
\hline DawaPlus $^{\circledR} 2.0$ & Alpha-cyperméthrine incorporé dans du polyéthylène & Intérimaire & Publié \\
\hline Duranet $^{\circledR}$ & Polyester enduit d'alpha-cyperméthrine & Intérimaire & Publié \\
\hline Interceptor ${ }^{\circledR}$ & Deltaméthrine incorporé dans du polypropylène & Complète & Publié \\
\hline LifeNet ${ }^{\circledR}$ & Alpha-cyperméthrine incorporé dans du polyéthylène & Intérimaire & Publié \\
\hline MAGNet $^{\mathrm{TM}}$ & Deltaméthrine incorporé dans du polyéthylène & Intérimaire & Publié \\
\hline Netprotect $^{\circledR}$ & Perméthrine incorporé dans du polyéthylène & Intérimaire & Publié \\
\hline Olyset $^{\circledR}$ & Perméthrine et PBO incorporés dans du polyéthylène & Complète & Publié \\
\hline Olyset ${ }^{\circledR}$ Plus & Polyester enduit de deltaméthrine & Intérimaire & En cours \\
\hline PermaNet $^{\circledR} 2.0$ & Polyester enduit de deltaméthrine avec bordures renforcées & Complète & Publié \\
\hline PermaNet $^{\circledR} 2.5$ & $\begin{array}{l}\text { Combinaison de polyester enduit de deltaméthrine avec bordures } \\
\text { renforcées (panneaux latéraux) et de deltaméthrine et PBO } \\
\text { incorporés dans du polyéthylène (toit) }\end{array}$ & Intérimaire & Publié \\
\hline PermaNet ${ }^{\circledR} 3.0$ & Alpha-cyperméthrine incorporé dans du polyéthylène & Intérimaire & Publié \\
\hline Royal Sentry ${ }^{\circledR}$ & Polyester enduit de deltaméthrine & Intérimaire & Publié \\
\hline Yorkool $^{\circledR} \mathrm{LN}$ & Deltamethrin coated on polyester & Complète & Publié \\
\hline
\end{tabular}

REMARQUE :

1. Les rapports des réunions du Groupe de travail WHOPES devraient être consultés pour des conseils détaillés sur l'utilisation et les recommandations. Ces rapports sont disponibles sur la page d'accueil de l'OMS sur I'Internet à l'adresse: www.who.int/whopes/recommendations/wgm/en; et

2. Les recommandations de l'OMS sur l'utilisation des pesticides en santé publique sont valides seulement lorsqu'elles sont liées aux spécifications de contrôle de la qualité de l'OMS. Les spécifications de l'OMS pour les pesticides en santé publique sont disponibles sur la page d'accueil de l'OMS sur I'Internet à : www.who.int/whopes/quality/newspecif/en

3. Pour une liste à jour, consulter le site Web de WHOPES (www.who.int/whopes).

SOURCE : Programme Pesticide Evaluation Scheme (WHOPES) de l'Organisation mondiale de la santé (61). 


\section{Références}

(1) Krzywinski, J., O. G. Grushko et N. J. Besansky. «Analysis of the complete mitochondrial DNA from Anopheles funestus: an improved dipteran mitochondrial genome annotation and a temporal dimension of mosquito evolution », dans $\mathrm{Mol}$ Phylogenet Evol, 2006, 39(2), p. 417-423

(2) Comité consultatif de la médecine tropicale et de la médecine des voyages (CCMTMV). «Déclaration sur la dengue. Déclaration d'un comité consultatif (DCC) », dans RMTC, 2009, vol. 35 (DCC-2), p. 1-12

(3) Kiszewski, A., A. Mellinger, A. Spielman, P. Malaney, S. E. Sachs et J. Sachs. "A global index representing the stability of malaria transmission », dans Am J Trop Med Hyg, 2004, vol. 70, no 5, p. 486-498

(4) Pates, H., et C. Curtis. «Mosquito behavior and vector control », dans Annu Rev Entomol, 2005, no 50, p. $53-70$

(5) Klun, A., D. Strickman, E. Rowton, J. Williams, M. Kramer, D. Roberts et coll. «Comparative resistance of Anopheles albimanus and Aedes aegypti to $\mathrm{N}, \mathrm{N}$-diethyl-3-methylbenzamide (Deet) and 2-methylpiperidinyl-3-cyclohexen-1-carboxamide (Al3-37220) in laboratory human-volunteer repellent assays », dans J Med Entomol, 2004, vol. 41, no 3, p. $418-422$

(6) Moore, S. J., et M. Debboun. « History of insect repellents », dans M. Debboun, S. Francis et D. Strickman (sous la dir. de), Insect repellents: Principles, methods and uses, CRC Press, 2007, p. 3-29

(7) Maia, M. F., et S. J. Moore. «Plant-based insect repellents: a review of their efficacy, development and testing », dans Malar J, 2011, no 10, suppl 1, p. S11

(8) Kitchen, L. W., K. L. Lawrence et R. E. Coleman. «The role of the United States military in the development of vector control products, including insect repellents, insecticides, and bed nets ", dans J Vector Ecol, 2009, vol. 34 , no 1 , p. 50-61

(9) Goodyer, L. I., A. M. Croft, S. P. Frances, N. Hill, S. J. Moore, S. P. Onyango et coll. « Expert review of the evidence base for arthropod bite avoidance ", dans J Travel Med, 2010, vol. 17, no 3, p. 182-192
(10) Comité consultatif de la médecine tropicale et de la médecine des voyages (CCMTMV). "Une approche de la médecine fondée sur les preuves », dans RMTC, 1994, vol. 20, p. 145-147

(11) Comité consultatif de la médecine tropicale et de la médecine des voyages (CCMTMV). « Déclaration relative aux mesures de protection individuelle pour prévenir les piqûres et les morsures d'arthropodes", dans RMTC, 2005, vol. 31 (DCC-4)

(12) Literature retrieval system [Internet]. United States: Armed Forces Pest Management Board. c1962- [recensé le 24 avril 2011]. Disponible à www.afpmb.org

(13) Croft, A. M. «Malaria: prevention in travellers», dans Clin Evid (en ligne), 2010, [pii] : 0903

(14) Balshem, H., M. Helfand, H. J. Schunemann, A. D. Oxman, R. Kunz, J. Brozek et coll. «GRADE guidelines: 3 . Rating the quality of evidence $»$, dans J Clin Epidemiol, 2011, vol. 64, no 4, p. 401-406

(15) Agence de réglementation de la lutte antiparasitaire [page d'accueil dans Internet], 2011 [recensé le 24 avril 2011]. Disponible à www.hc-sc.gc.ca/cps-spc/ pest/index-fra.php

(16) United States Environmental Protection Agency. Office of Pesticide Programs [page d'accueil dans Internet], 2011 [recensé le 24 avril 2011], Disponible à www.epa.gov/pesticides

(17) DjËnontin, A., J. Chabi, T. Baldet, S. Irish, C. Pennetier, J. M. Hougard et coll. « Managing insecticide resistance in malaria vectors by combining carbamatetreated plastic wall sheeting and pyrethroid-treated bed nets ", dans Malar J, 2009, 8:233

(18) Ranson, H., R. N'guessan, J. Lines, N. Moiroux, Z. Nkuni et V. Corbel. «Pyrethroid resistance in African anopheline mosquitoes: what are the implications for malaria control? ", dans Trends Parasitol, 2011, vol. 27, no 2, p. 91-98

(19) Comité consultatif de la médecine tropicale et de la médecine des voyages (CCMTMV). «Recommandations canadiennes pour la prévention et le traitement du paludisme (malaria) chez les voyageurs internationaux ", dans RMTC, 2009, vol. 35 , suppl 1, p. 1-82 
(20) Lindsay, S. W., P. M. Emerson et J. D. Charlwood. "Reducing malaria by mosquito-proofing houses", dans Trends Parasitol, 2002, vol. 18, no 11, p. 510-514

(21) Lindsay, S. W., M. Jawara, K. Paine, M. Pinder, G. E. Walraven et P. M. Emerson. « Changes in house design reduce exposure to malaria mosquitoes ", dans Trop Med Int Health, 2003, vol. 8, no 6, p. 512-517.

(22) Kirby, M. J., D. Ameh, C. Bottomley, C. Green, M. Jawara, P. J. Milligan et coll. " Effect of two different house screening interventions on exposure to malaria vectors and on anaemia in children in The Gambia: a randomised controlled trial », dans Lancet, 2009, vol. 374, no 9694, p. 998-1009

(23) Njie, M., E. Dilger, S. W. Lindsay et M. J. Kirby. "Importance of eaves to house entry by anopheline, but not culicine, mosquitoes", dans J Med Entomol, 2009, vol. 46, no 3, p. 505-510

(24) Chappuis, F., S. K. Sharma, N. Jha, L. Loutan et P. A. Bovier. "Protection against snake bites by sleeping under a bed net in southeastern Nepal », dans Am J Trop Med Hyg, 2007, vol. 77, no 1. p. 197-199

(25) Christophers, S. « Mosquito repellents; being a report of the work of the mosquito repellent inquiry, Cambridge, 1943-5 », dans J Hyg, 1947, vol. 45, no 2 p. 176-231

(26) Schoepke, A., F. Steffen et G. Norman. «Effectiveness of personal protection measures against mosquito bites for malaria prophylaxis in travelers $»$, dans J Travel Med, décembre 1998, vol. 5, no 4, p. 182-192

(27) Joy, R. J. " Malaria in American troops in the South and Southwest Pacific in World War II ", dans Med Hist, 1999, vol. 43, no 2, p. 192-207

(28) Wormser, G. P., R. J. Dattwyler, E. D. Shapiro, J. J. Halperin, A. C. Steere, M. S. Klempner et coll. "The clinical assessment, treatment, and prevention of lyme disease, human granulocytic anaplasmosis, and babesiosis: clinical practice guidelines by the Infectious Diseases Society of America », dans Clin Infect Dis, 2006, vol. 43, no 9, p. 1089-1134

(29) Agence de la santé publique du Canada. Maladie de Lyme - Fiche de renseignements [Internet], 2010 [recensé le 24 avril 2011]. Disponible à www.phac-aspc.gc.ca/id-mi/lyme-fs-fra.php
(30) Syed, Z., et W. S. Leal. « Mosquitoes smell and avoid the insect repellent DEET », dans Proc Natl Acad Sci USA, 2008, vol. 105, no 36, p. 13598-13603

(31) Liu, C., R. J. Pitts, J. D. Bohbot, P. L. Jones, G. Wang et L. J. Zwiebel. « Distinct olfactory signaling mechanisms in the malaria vector mosquito Anopheles gambiae », dans PLoS Biol, 2010, vol. 8, no 8

(32) Bohbot, J. D., et J. C. Dickens. « Insect repellents: Modulators of mosquito odorant receptor activity ", dans PLoS One, 2010, vol. 5, no 8, p. 12138

(33) United States Environmental Protection Agency. Office of Chemical Safety and Pollution Prevention. Product performance test guidelines: Insect repellents to be applied to human skin [Internet], 2010 [recensé le 24 avril 2011]. Rapport no OPPTS 810.3700. Disponible à www.epa.gov/hsrb/files/ meeting-materials/jun-23-2010-public-meeting/ Repellent_Guideline_5-13-2010\%5B1\%5D.pdf

(34) Hill, N., A. Lenglet, A. M. Arnez et I. Carneiro. "Plant based insect repellent and insecticide treated bed nets to protect against malaria in areas of early evening biting vectors: double blind randomised placebo controlled clinical trial in the Bolivian Amazon », dans BMJ, 2007, vol. 335, no 7628, p. 1023

(35) Centers for Disease Control and Prevention. Updated information regarding insect repellents [Internet], 2008 [recensé le 24 avril 2011]. Disponible à www.cdc.gov/ncidod/dvbid/westnile/ RepellentUpdates.htm

(36) Zielinski-Gutierrez, E., R. A Wirtz et R. S. Nasci. «The Pre-Travel Consultation. Protection against mosquitoes, ticks, and other insects and arthropods", dans CDC health information for international travel 2012. Atlanta, U.S. Department of Health and Human Services, Public Health Service, 2011, p. 86-89

(37) Organisation mondiale de la santé. WHO Pesticide Evaluation Scheme [page d'accueil dans Internet], 2011 [recensé le 24 avril 2011]. Disponible à www.who.int/whopes/en

(38) Organisation mondiale de la santé. WHO Pesticide Evaluation Scheme. Report of the fourth WHOPES working group meeting: review of: IR3535; KBR3023; (RS)-methoprene 20\% EC, pyriproxyfen 0.5\% GR; and lambda-cyhalothrin 2.5\% CS [Internet], 2001 [recensé le 24 avril 2011]. Rapport no WHO/CDS/ WHOPES/2001.2. Disponible à http://apps.who.int/ ctd/whopes/docs/Report4thWHOPESWGM.pdf 
(39) Agence de réglementation de la lutte antiparasitaire. Projet d'acceptabilité d'homologation continue:

Réévaluation de l'huile de citronnelle et des composés apparentés pour utilisation comme insectifuge personnel, Ottawa, Santé Canada, 2004, Rapport no PACR2004-36

(40) Fradin, M. S., et J. F. Day. « Comparative efficacy of insect repellents against mosquito bites », dans N Engl J Med, 2002, vol. 347, no 1, p. 13-18

(41) Agence de réglementation de la lutte antiparasitaire, Division de la documentation et de la coordination des demandes d'homologation. Décision de réévaluation: Insectifuges corporels contenant du DEET (N,N-diéthyl-m-toluamide et composés apparentés) [Internet], Ottawa, Santé Canada, le 15 avril 2002 [recensé le 24 avril 2011]. Rapport no RRD2002-01. Disponible à http://dsp-psd.pwgsc. gc.ca/Collection/H113-12-2002-1F.pdf

(42) Schofield, S., M. Tepper et R. Gadawski. « Laboratory and field evaluation of the impact of exercise on the performance of regular and polymer-based deet repellents », dans J Med Entomol, 2007, vol. 44, no 6, p. 1026-1031

(43) Barnard, D., et R. Xue. «Biometrics and behaviour in mosquito repellent assays ", dans M. Debboun, S. Francis et D. Strickman (sous la dir. de), Insect repellents: Principles, methods and uses, CRC Press, 2007, p. 111-124

(44) Granett, P. «Studies of mosquito repellents, I. Test procedure and method of evaluating test data ", dans J Econ Entomol, 1940, vol. 33, no 3, p. 563-565

(45) Vickery, J. P., D. R. Tribble, S. D. Putnam, T. McGraw, J. W. Sanders, A. W. Armstrong et coll. «Factors associated with the use of protective measures against vector-borne diseases among troops deployed to Iraq and Afghanistan », dans Mil Med, 2008, vol. 173, no 11, p. 1060-1067

(46) Keystone, J. S. « Compliance with travel health recommendations ", dans H. I Dupont et F. Steffen (sous la dir. de), Textbook of travel medicine and health, Londres, B. C. Decker Inc., 2001

(47) Alon, D., P. Shitrit et M. Chowers. « Risk behaviors and spectrum of diseases among elderly travelers: A comparison of younger and older adults », dans J Travel Med, 2010, vol. 17, no 4, p. 250-255
(48) Webb, C. E., et R. C. Russell. «Insect repellents and sunscreen: implications for personal protection strategies against mosquito-borne disease ", dans Aust N Z J Public Health, 2009, vol. 33, no 5, p. $485-490$

(49) Montemarano, A. D., R. K. Gupta, J. R. Burge et K. Klein. "Insect repellents and the efficacy of sunscreens », dans Lancet, 1997, vol. 349, no 9066, p. $1670-1671$

(50) Chen, T., F. J. Burczynski, D. W. Miller et X. Gu. «Percutaneous permeation comparison of repellents picaridin and DEET in concurrent use with sunscreen oxybenzone from commercially available preparations », dans Pharmazie, 2010, vol. 65, no 11, p. 835-839

(51) Wang, T., et X. Gu. « In vitro percutaneous permeation of the repellent DEET and the sunscreen oxybenzone across human skin », dans J Pharm Pharm Sci, 2007, vol. 10, no 1, p. 17-25

(52) Gu, X., T. Wang, D. M. Collins, S. Kasichayanula et F. J. Burczynski. «In vitro evaluation of concurrent use of commercially available insect repellent and sunscreen preparations », dans Br J Dermatol, 2005, vol. 152, no 6, p. 1263-1267

(53) Lengeler, C. «Insecticide-treated bed nets and curtains for preventing malaria », dans Cochrane Database Syst Rev, 2004, no 2, CD000363

(54) Gamble, C., J. P. Ekwaru et F. O. ter Kuile. "Insecticide-treated nets for preventing malaria in pregnancy ", dans Cochrane Database Syst Rev, 2006, no 2, CD003755

(55) Pluess, B., F. C. Tanser, C. Lengeler et B. L. Sharp. "Indoor residual spraying for preventing malaria », dans Cochrane Database Syst Rev, 2010, no 4, CD006657

(56) Organisation mondiale de la santé. WHO Pesticide Evaluation Scheme. Guidelines for testing mosquito adulticides for indoor residual spraying and treatment of mosquito nets [Internet], 2006 [recensé le 24 avril 2011]. Rapport no WHO/CDS/NTD/WHOPES/ GCDPP/2006.3. Disponible à http://whqlibdoc.who. int/hq/2006/WHO_CDS_NTD_WHOPES_ GCDPP_2006.3_eng.pdf 
(57) Organisation mondiale de la santé. WHO Pesticide Evaluation Scheme. WHO recommended insecticides for indoor residual spraying against malaria vectors [Internet], 2009 [mise à jour en octobre 2009; recensé le 24 avril 2011]. Disponible à www.who.int/whopes/ Insecticides_IRS_Malaria_09.pdf

(58) Organisation mondiale de la santé. WHO Pesticide Evaluation Scheme. A generic risk assessment model for insecticide treatment and subsequent use of mosquito nets [Internet], 2004 [recensé le 24 avril 2011]. Rapport no WHO/CDS/WHOPES/ GCDPP/2004.6; WHO/PCS/04.1. Disponible à http://whqlibdoc.who.int/hq/2004/WHO_PCS_04.1.pdf

(59) Organisation mondiale de la santé. WHO Pesticide Evaluation Scheme. WHO recommended insecticide products treatment of mosquito nets for malaria vector control [Internet], 2011 [mis à jour en décembre 2007; recensé le 18 avril 2011]. Disponible à www.who.int/ whopes/Insecticides_ITN_Malaria_ok3.pdf

(60) Organisation mondiale de la santé. WHO Pesticide Evaluation Scheme. WHO specifications for pesticides used in public health [Internet], 2011 [recensé le 24 avril 2011]. Disponible à www.who.int/whopes/quality/ newspecif/en

(61) Organisation mondiale de la santé. WHO Pesticide Evaluation Scheme. WHO recommended long-lasting insecticidal mosquito nets [Internet], 2012 [mis à jour en juillet 2012; recensé le 9 juillet 2012]. Disponible à www.who.int/whopes/Long_lasting_insecticidal_nets_ Jul_2012.pdf

(62) Organisation mondiale de la santé. WHO Pesticide Evaluation Scheme. Guidelines for laboratory and field testing of long-lasting insecticidal mosquito nets [Internet], 2005 [recensé le 24 avril 2011]. Rapport no WHO/CDS/WHOPES/GCDPP/2005.11. Disponible à http://whqlibdoc.who.int/hq/2005/WHO_CDS_ WHOPES_GCDPP_2005.11.pdf

(63) Organisation mondiale de la santé. WHO Pesticide Evaluation Scheme. Instructions for treatment and use of insecticide-treated mosquito nets [Internet], 2002 [recensé le 24 avril 2011]. Disponible à www.who.int/ malaria/publications/atoz/who_cds_rbm_2002_41/en

(64) Schreck, C. E., K. Posey et D. Smith. «Durability of permethrin as a potential clothing treatment to protect against blood-feeding arthropods ", dans J Econ Entomol, 1978, vol. 71, no 3, p. 397-400
(65) Schreck, C. E., D. A. Carlson, D. E. Weidhaas, K. Posey et D. Smith. "Wear and aging tests with permethrintreated cotton-polyester fabric », dans J Econ Entomol, 1980, vol. 73, no 3, p. 451-453

(66) Schreck, C. E., D. G. Haile et D. L. Kline. «The effectiveness of permethrin and deet, alone or in combination, for protection against Aedes taeniorhynchus », dans Am J Trop Med Hyg, 1984, vol. 33, no 4, p. 725-730

(67) Vaughn, M. F., et S. R. Meshnick. «Pilot study assessing the effectiveness of long-lasting permethrinimpregnated clothing for the prevention of tick bites ", dans Vector Borne Zoonotic Dis, 2011 [prépublication de Epub]

(68) Soto, J., F. Medina, N. Dember et J. Berman. «Efficacy of permethrin-impregnated uniforms in the prevention of malaria and leishmaniasis in Colombian soldiers", dans Clin Infect Dis, 1995, vol. 21, no 3, p. 599-602

(69) Ministère de la Justice du Canada. Règlement sur les produits antiparasitaires. DORS/2006-124. 2006 [mis à jour le 3 juin 2010; recensé le 24 avril 2011]. Disponible à http://lois-laws.justice.gc.ca/PDF/DORS-2006-124.pdf

(70) Rowland, M., T. Freeman, G. Downey, A. Hadi et M. Saeed. " DEET mosquito repellent sold through social marketing provides personal protection against malaria in an area of all-night mosquito biting and partial coverage of insecticide-treated nets: a casecontrol study of effectiveness », dans Trop Med Int Health, 2004, vol. 9, no 3, p. 343-350

(71) Welt, L. G. « Use of dimethylphthalate impregnated clothing as protection against scrub typhus », dans Am J Trop Med Hyg, mars 1947, vol. 27, no 2, p. 221-224

(72) Gladwell, M. «Fred Soper and the global malaria eradication programme ", dans J Public Health Policy, 2002, vol. 23, no 4, p. 479-497

(73) Patterson, R. « Dr. William Gorgas and his war with the mosquito ", dans CMAJ, 1989, vol. 141, no 6, p. 596-597 et 599

(74) Rowland, M., N. Durrani, S. Hewitt, N. Mohammed, M. Bouma, I. Carneiro et coll. « Permethrin-treated chaddars and top-sheets: appropriate technology for protection against malaria in Afghanistan and other complex emergencies ", dans Trans $R$ Soc Trop Med Hyg, 1999, vol. 93, no 5, p. 465-472 
(75) Surgeoner, G. A., et B. V. Helson. «A field evaluation of electrocutors for mosquito control in southern Ontario », dans Proc Entomol Soc Ontario, 1977, vol. 108 , p. 53-57

(76) Enayati, A. A., J. Hemingway et P. Garner. « Electronic mosquito repellents for preventing mosquito bites and malaria infection », dans Cochrane Database Syst Rev, 2007, no 2, CD005434

(77) Nasci, R. S., C. W. Harris et C. K. Porter. « Failure of an insect electrocuting device to reduce mosquito biting », dans Mosquito News, 1983, vol. 43, no 2, p. $180-184$

(78) Jensen, T., R. Lampman, M. C. Slamecka et R. J. Novak. «Field efficacy of commercial antimosquito products in Illinois », dans J Am Mosq Control Assoc, 2000, vol. 16, no 2, p. 148-152

(79) Burkett, D. A., W. J. Lee, K. W. Lee, H. C. Kim, H. I. Lee, J. S. Lee et coll. «Light, carbon dioxide, and octenolbaited mosquito trap and host-seeking activity evaluations for mosquitoes in a malarious area of the Republic of Korea ", dans J Am Mosq Control Assoc, 2001, vol. 17, no 3, p. 196-205

(80) Kitau, J., H. Pates, T. R. Rwegoshora, D. Rwegoshora, J. Matowo, E. J. Kweka et coll. «The effect of Mosquito Magnet Liberty Plus trap on the human mosquito biting rate under semi-field conditions ", dans J Am Mosq Control Assoc, 2010, vol. 26, no 3, p. 287-294

(81) Matsuda, B. M., G. A. Surgeoner, J. D. Heal, A. O. Tucker et M. J. Maciarello. « Essential oil analysis and field evaluation of the citrosa plant "Pelargonium citrosum" as a repellent against populations of Aedes mosquitoes ", dans J Am Mosq Control Assoc, mars 1996, vol. 12, no 1, p. 69-74
(82) Cilek, J. E., et E. T. Schreiber. «Failure of the "mosquito plant", Pelargonium x citrosum 'van Leenii', to repel adult Aedes albopictus and Culex quinquefasciatus in Florida », dans J Am Mosq Control Assoc, 1994, vol. 10, no 4, p. 473-476

(83) Khan, A. A., H. I. Maibach, W. G. Strauss et W. R. Fenley. "Vitamin B1 is not a systemic mosquito repellent in man », dans Trans St Johns Hosp Dermatol Soc, 1969, vol. 55, no 1, p. 99-102

(84) Durden, L. A., et G. R. Mullen (sous la dir. de). Medical and Veterinary Entomology, New York, Academic Press, 2002

(85) Défense nationale Canada. Anthropod-borne Disease Surveillance Manual. GDG Environnement, 2006

(86) Rozendaal, J. A. Vector control: methods for use by individuals and communities. Genève, 1977

(87) Personal Protection Against Vectors working groups. "Personal protection against biting insects and ticks", dans Parasite, 2011, no 18, p. 93-111

(88) Société de Médecine des Voyages et Société Française de Parasitologie. Recommandations de bonne pratique : Protection personnelle antivectorielle - texte court, 2010 [recensé le 6 mai 2011]. Disponible à www.medecine-voyages.fr/ publications/ppavtextecourt.pdf 